\title{
ON STRICHARTZ ESTIMATES FOR SCHRÖDINGER OPERATORS IN COMPACT MANIFOLDS WITH BOUNDARY
}

\author{
MATTHEW D. BLAIR, HART F. SMITH, AND CHRISTOPHER D. SOGGE
}

(Communicated by Andreas Seeger)

\begin{abstract}
We prove local Strichartz estimates with a loss of derivatives over compact manifolds with boundary. Our results also apply more generally to compact manifolds with Lipschitz metrics.
\end{abstract}

\section{INTRODUCTION}

Let $(M, \mathrm{~g})$ be a Riemannian manifold of dimension $n \geq 2$. Strichartz estimates are a family of dispersive estimates on solutions $u(t, x):[-T, T] \times M \rightarrow \mathbb{C}$ to the Schrödinger equation

$$
D_{t} u+\Delta_{\mathrm{g}} u=0, \quad u(0, x)=f(x)
$$

where $\Delta_{\mathrm{g}}$ denotes the Laplace-Beltrami operator on $(M, \mathrm{~g})$ and $D_{t}=i^{-1} \partial_{t}$. In their most general form, local Strichartz estimates state that

$$
\|u\|_{L^{p}\left([-T, T] ; L^{q}(M)\right)} \leq C\|f\|_{H^{s}(M)}
$$

where $H^{s}$ denotes the $L^{2}$ Sobolev space over $M$, and $2 \leq p, q \leq \infty$ satisfy

$$
\frac{2}{p}+\frac{n}{q}=\frac{n}{2}, \quad(n, p, q) \neq(2,2, \infty) .
$$

Such estimates are well established in flat Euclidean space, where $M=\mathbb{R}^{n}$ and $\mathrm{g}_{i j}=$ $\delta_{i j}$. In that case $s=0$, and one can take $T=\infty$; see for example Strichartz [11], Ginibre and Velo [5], Keel and Tao [6], and the references therein. Estimates for the standard flat 2-torus were shown by Bourgain [2] to hold for any $s>0$.

There is also considerable interest in developing these estimates for non-flat geometries, and also for compact domains. In the case where $M$ is compact and $\partial M=\varnothing$, Burq, Gérard, and Tzvetkov [3] established (1.2) with $s=\frac{1}{p}$. Hence there is a loss of derivatives in their estimate when compared to the case of flat geometries.

A simple investigation of the Schrödinger evolution on spherical harmonics where $M=\mathbb{S}^{n}$ shows that some loss of derivatives must occur. For instance, with $n=2$, by taking the initial data to be a highest weight spherical harmonic on $\mathbb{S}^{2}$ one

Received by the editors October 31, 2006.

2000 Mathematics Subject Classification. Primary 35Q40, 35B65; Secondary 35Q55, 35A17.

The authors were supported by the National Science Foundation, Grants DMS-0140499, DMS0099642 , and DMS-0354668. 
concludes that the best possible local $L_{x}^{2} \rightarrow L_{t, x}^{4}$ bounds would involve a loss of $1 / 8$ derivatives. This sharp estimate and related ones for Zoll surfaces were obtained in [3]. It is not known, however, whether the weaker estimates involving a loss of $\frac{1}{p}$ derivatives in [3] for general compact manifolds without boundary can be improved.

In the case where $\partial M \neq \varnothing$, one also considers Dirichlet or Neumann boundary conditions in addition to (1.1):

$$
\left.u(t, x)\right|_{\partial M}=0 \text { (Dirichlet), }\left.\quad N_{x} \cdot \nabla u(t, x)\right|_{\partial M}=0 \text { (Neumann) }
$$

where $N_{x}$ denotes the unit normal vector field to $\partial M$. Here one expects a further loss of derivatives in the estimates. The Rayleigh whispering gallery modes over the unit disk in $\mathbb{R}^{2}$ provide examples of Dirichlet eigenfunctions that accumulate their energy near the boundary, contributing to high $L^{p}$ norms. Applying the Schrödinger evolution to these eigenfunctions shows that $s \geq \frac{1}{6}$ is necessary for the Strichartz estimate with $p=q=4$. Recently, Anton [1] showed that the estimates (1.2) hold on general manifolds with boundary provided $s>\frac{3}{2 p}$. In addition, the arguments of [1] work equally well for a manifold $M$ without boundary equipped with a metric $\mathrm{g}$ of Lipschitz regularity.

In this work, we improve on the current results for compact $(M, \mathrm{~g})$ where either $\partial M \neq \varnothing$, or $\partial M=\varnothing$ and $\mathrm{g}$ is Lipschitz, by showing that Strichartz estimates hold with a loss of fewer derivatives.

Theorem 1.1. Let $(M, \mathrm{~g})$ be either a smooth compact Riemannian manifold with boundary, or a manifold without boundary equipped with a Lipschitz metric g. Then the following Strichartz estimate holds for any Strichartz pair (1.3):

$$
\left\|e^{i t \Delta_{\mathrm{g}}} f\right\|_{L^{p}\left([-T, T] ; L^{q}(M)\right)} \lesssim\|f\|_{H^{\frac{4}{3 p}}(M)} .
$$

In the case where $(M, \mathrm{~g})$ is a boundaryless manifold with $\mathrm{g} \in C^{\infty}$, the estimate of Burq-Gérard-Tzvetkov (1.2) with $s=\frac{1}{p}$, while not known to be sharp, is a natural result by the following heuristic argument. For a general compact manifold, there are no conjugate points for the geodesic flow at distance less than the injectivity radius of the manifold. Given a solution to the Schrödinger equation whose frequencies are concentrated at $\lambda$, energy propagates at speed $\approx \lambda$. Hence, a frequency $\lambda$ solution should possess good dispersive properties at least until time $T_{\lambda} \approx \frac{1}{\lambda}$. We thus expect to be able to prove a Strichartz estimate with no loss of derivatives for such a solution over a time interval of size roughly $\frac{1}{\lambda}$. By considering a sum over such intervals we should obtain a Strichartz estimate over a time interval of unit size, only with a constant appearing on the right-hand side that is a constant multiple of $\lambda^{\frac{1}{p}}$. This corresponds to $s=\frac{1}{p}$ in the estimate, and Littlewood-Paley theory yields the estimate for arbitrary solutions.

In the case where $\partial M \neq \varnothing$, the boundary conditions affect the flow of energy near the boundary. A key strategy involves reflecting the metric and the solution across the boundary to obtain a Schrödinger equation on a manifold without boundary, but with a metric that has Lipschitz singularities along $\partial M$. Hence matters reduce to considering the Schrödinger evolution for Lipschitz metrics. In this case, when establishing estimates for solutions at frequency $\lambda$, one can replace the rough metric by a regularized metric which has conjugate points at distance roughly $\lambda^{-\frac{1}{3}}$ apart. Therefore, the solutions should possess good dispersive properties over a time interval of size roughly $\lambda^{-\frac{4}{3}}$. This now yields a Strichartz estimate over a time interval of unit size with a loss of $\frac{4}{3 p}$ derivatives. Hence, for manifolds with 
boundary, (1.4) appears to be the natural analog of the aforementioned estimates of [3] for the general boundaryless case.

Our proof of Theorem 1.1 follows the above heuristics. In section 2 the solution is localized spatially and a coordinate chart is used to work on $\mathbb{R}^{n}$; a LittlewoodPaley decomposition then reduce matters to establishing Strichartz estimates for components of the solutions dyadically localized in frequency. As alluded to above, we then seek to prove Strichartz estimates with no loss of derivatives over time intervals of size $\lambda^{-\frac{4}{3}}$ for components of the solution localized at frequency $\lambda$. This involves regularizing the metric by truncating its frequency to a scale dependent on $\lambda$. Rescaling the solution then reduces the problem to establishing Strichartz estimates for metrics with 2 bounded derivatives over small time intervals whose size also depends on the frequency. Section 3 uses a phase space transform to construct a parametrix for such Schrödinger operators, and section 4 concludes the paper by showing that the parametrix yields the desired estimates.

Notation. In what follows $d$ will denote the gradient operator that maps scalar functions to vector fields and vector fields to matrix functions in the natural way. The expression $X \lesssim Y$ means that $X \leq C Y$ for some $C$ depending only on $n$ and on the Lipschitz norm of the metric.

\section{Reductions}

We will establish Theorem 1.1 more generally for operators on $M$ that take the following form in local coordinates:

$$
(P f)(x)=\rho(x)^{-1} \sum_{i, j=1}^{n} \partial_{i}\left(\rho(x) \mathrm{g}^{i j}(x) \partial_{j} f(x)\right) .
$$

Such an operator is selfadjoint in the measure $d \mu=\rho(x) d x$. Neumann conditions and the boundary normal are defined with respect to the metric $g_{i j}$.

We start by reducing the case of a manifold $M$ with boundary and $P$ smooth to the case of a compact manifold $M$ without boundary, with $P$ having coefficients of Lipschitz regularity. For this, let $\tilde{M}$ denote the double of $M$, identified along $\partial M$. We define a differentiable structure on $\tilde{M}$ near $\partial M$ using geodesic normal coordinates in $\mathrm{g}_{i j}$, so $x_{n}>0$ and $x_{n}<0$ define the two copies of $M$. In these coordinates, $\mathrm{g}^{n i}=0$ for $i \neq n$; hence $P$ contains no cross terms between $\partial_{n}$ and $\partial_{i}$. The operator $\tilde{P}$ with coefficients $\mathrm{g}^{i j}\left(x^{\prime},\left|x_{n}\right|\right)$ and $\rho\left(x^{\prime},\left|x_{n}\right|\right)$ is thus symmetric under $x_{n} \rightarrow-x_{n}$, and extends the lift of $P$ to $\tilde{M}$ across $\partial M$ to one with Lipschitz coefficients. Eigenspaces for $\tilde{P}$ decompose into symmetric and antisymmetric functions; these correspond to extensions of eigenfunctions for $P$ satisfying Dirichlet (resp. Neumann) conditions, and each eigenfunction is of regularity $C^{1,1}$ across the boundary. The Schrödinger flow for $\tilde{P}$ is thus easily seen to extend that for $P$, and the Strichartz estimates for $P$ follow by establishing such estimates for $\tilde{P}$ on $\tilde{M}$.

We assume henceforth that $M$ is a compact manifold with smooth differentiable structure, on which an operator $P$ of the form (2.1) is given, with coefficients of Lipschitz regularity. Define $L^{q}$-Sobolev spaces on $M$ using the spectral resolution of $P$,

$$
\|f\|_{W^{s, q}(M)}=\left\|\left\langle D_{P}\right\rangle^{s} f\right\|_{L^{q}(M)}, \quad\left\langle D_{P}\right\rangle=(1-P)^{\frac{1}{2}} .
$$

When $q=2$ we denote $W^{s, q}$ by $H^{s}$. By elliptic regularity (e.g. [4, Theorem 8.10] for $q=2$, and [4, Theorem 9.11] or [14, $\S 2.2]$ for other $q$ ) the spaces $W^{s, q}$ 
for $1<q<\infty$ coincide with the Sobolev spaces defined using local coordinates, provided $0 \leq s \leq 2$.

Suppose that $u(t, x)=\left(e^{i t P} f\right)(x)$. Then we need to establish

$$
\|u\|_{L^{p}\left([-T, T] ; W^{s, q}(M)\right)} \lesssim\|f\|_{H^{1}(M)}, \quad s=1-\frac{4}{3 p} .
$$

Let $u=\sum_{k=0}^{\infty} u_{k}$ denote a Littlewood-Paley partition of $u$, where $u_{k}$ for $k \geq 1$ is spectrally localized to $\left\langle D_{P}\right\rangle \approx 2^{k}$. Then, for $p, q \geq 2$,

$$
\left\|\left\langle D_{P}\right\rangle^{s} u\right\|_{L_{t}^{p} L_{x}^{q}} \approx\left\|\left(\left\langle D_{P}\right\rangle^{s} u\right)_{k}\right\|_{L_{t}^{p} L_{x}^{q} \ell_{k}^{2}} \leq\left\|\left\langle D_{P}\right\rangle^{s} u_{k}\right\|_{\ell_{k}^{2} L_{t}^{p} L_{x}^{q}}
$$

and

$$
\|f\|_{H^{1}(M)} \approx\left\|f_{k}\right\|_{\ell_{k}^{2} H^{1}(M)}
$$

hence it suffices to show, uniformly over $k$, that

$$
\left\|u_{k}\right\|_{L^{p}\left([-T, T] ; W^{s, q}(M)\right)} \lesssim\left\|f_{k}\right\|_{H^{1}(M)}, \quad s=1-\frac{4}{3 p} .
$$

By taking a finite partition of unity, it suffices to prove that

$$
\left\|\psi u_{k}\right\|_{L^{p}\left([-T, T] ; W^{s, q}\left(\mathbb{R}^{n}\right)\right)} \lesssim\left\|u_{k}\right\|_{L^{\infty}\left([-T, T] ; H^{1}(M)\right)}
$$

for each smooth cutoff $\psi$ supported in a suitably chosen coordinate chart. We will choose coordinate charts such that the image contains the unit ball, and

$$
\left\|\mathrm{g}^{i j}-\delta_{i j}\right\|_{\operatorname{Lip}\left(B_{1}(0)\right)} \leq c_{0}, \quad\|\rho-1\|_{\operatorname{Lip}\left(B_{1}(0)\right)} \leq c_{0},
$$

for $c_{0}$ taken to be suitably small. (This may require multiplying $\rho$ by a harmless constant.) We take $\psi$ supported in the unit ball, and assume $\mathrm{g}^{i j}$ and $\rho$ are extended so that the above holds globally on $\mathbb{R}^{n}$.

Let $\left\{\beta_{j}(D)\right\}_{j \geq 0}$ be a Littlewood-Paley partition of unity on $\mathbb{R}^{n}$, and let $v_{j}=$ $\beta_{j}(D) \psi u_{k}$. We will prove that, for each $j$,

$$
\left\|\langle D\rangle^{s} v_{j}\right\|_{L_{t}^{p} L_{x}^{q}} \lesssim\left\|v_{j}\right\|_{L_{t}^{\infty} H_{x}^{1}}+2^{-\frac{j}{3}}\left\|\left(D_{t}+P\right) v_{j}\right\|_{L_{t}^{\infty} L_{x}^{2}},
$$

with all norms taken over $[-T, T] \times \mathbb{R}^{n}$ and $\langle D\rangle=(1-\Delta)^{\frac{1}{2}}$.

This will imply (2.2), provided we dominate the sum over $j$ of the right-hand side of (2.3) by $\left\|u_{k}\right\|_{L^{\infty}\left([-T, T] ; H^{1}(M)\right)}$, which we now do.

For a Lipschitz function $a,\left[\beta_{j}(D), a\right]: H^{s-1} \rightarrow H^{s}$ for $s=0,1$. Hence $\left[P, \beta_{j}(D) \psi\right]: H^{1} \rightarrow L^{2}$, and it follows that

$$
\left\|\left(D_{t}+P\right) v_{j}\right\|_{L_{t}^{\infty} L_{x}^{2}} \lesssim\left\|u_{k}\right\|_{L_{t}^{\infty} H^{1}(M)}
$$

hence the second term on the right of (2.3) is bounded by a geometric series. For the first term, note that

$$
\begin{aligned}
\left\|v_{j}\right\|_{L_{t}^{\infty} H_{x}^{1}} & \lesssim \min \left(2^{j}\left\|v_{j}\right\|_{L_{t}^{\infty} L_{x}^{2}}, 2^{-j}\left\|v_{j}\right\|_{L_{t}^{\infty} H_{x}^{2}}\right) \\
& \lesssim \min \left(2^{j}\left\|u_{k}\right\|_{L_{t}^{\infty} L^{2}(M)}, 2^{-j}\left\|u_{k}\right\|_{L_{t}^{\infty} H^{2}(M)}\right) .
\end{aligned}
$$

The sum over $j$ is dominated by

$$
2^{k}\left\|u_{k}\right\|_{L_{t}^{\infty} L^{2}(M)}+2^{-k}\left\|u_{k}\right\|_{L_{t}^{\infty} H^{2}(M)} \lesssim\left\|u_{k}\right\|_{L_{t}^{\infty} H^{1}(M)} .
$$

Setting $\lambda=2^{j}$, and denoting $v_{j}$ by $v_{\lambda}$, the estimate (2.3) is equivalent to

$$
\begin{aligned}
\left\|v_{\lambda}\right\|_{L^{p}\left([-T, T] ; L^{q}\left(\mathbb{R}^{n}\right)\right)} & \\
& \lesssim \lambda^{\frac{4}{3 p}}\left\|v_{\lambda}\right\|_{L^{\infty}\left([-T, T] ; L^{2}\left(\mathbb{R}^{n}\right)\right)}+\lambda^{\frac{4}{3 p}-\frac{4}{3}}\left\|\left(D_{t}+P\right) v_{\lambda}\right\|_{L^{\infty}\left([-T, T] ; L^{2}\left(\mathbb{R}^{n}\right)\right)} .
\end{aligned}
$$

This, in turn, follows by showing that for any interval $I_{\lambda}$ of length $\lambda^{-\frac{4}{3}}$, we have

$$
\left\|v_{\lambda}\right\|_{L^{p}\left(I_{\lambda} ; L^{q}\left(\mathbb{R}^{n}\right)\right)} \lesssim\left\|v_{\lambda}\right\|_{L^{\infty}\left(I_{\lambda} ; L^{2}\left(\mathbb{R}^{n}\right)\right)}+\left\|\left(D_{t}+P\right) v_{\lambda}\right\|_{L^{1}\left(I_{\lambda} ; L^{2}\left(\mathbb{R}^{n}\right)\right)} .
$$


We now regularize the coefficients of $P$ by setting

$$
\mathrm{g}_{\lambda}^{i j}=S_{\lambda^{-2 / 3}}\left(\mathrm{~g}^{i j}\right), \quad \rho_{\lambda}=S_{\lambda^{-2 / 3}}(\rho),
$$

where $S_{\lambda^{2 / 3}}$ denotes a truncation of a function to frequencies less than $\lambda^{\frac{2}{3}}$, and let $P_{\lambda}$ denote the operator with coefficients $g_{\lambda}^{i j}$ and $\rho_{\lambda}$. Since $\left|g_{\lambda}^{i j}-g^{i j}\right| \lesssim \lambda^{-\frac{2}{3}}$, and similarly for $\rho$, it follows that

$$
\left\|\left(P-P_{\lambda}\right) v_{\lambda}\right\|_{L^{1}\left(I_{\lambda} ; L^{2}\left(\mathbb{R}^{n}\right)\right)} \lesssim\left\|v_{\lambda}\right\|_{L^{\infty}\left(I_{\lambda} ; L^{2}\left(\mathbb{R}^{n}\right)\right)},
$$

and we may thus replace $P$ by $P_{\lambda}$ on the right-hand side of $(2.4)$ without changing the estimate.

Finally, we rescale the problem. Let $\mu=\lambda^{\frac{2}{3}}$, and define

$$
\begin{aligned}
u_{\mu}(t, x) & =v_{\lambda}\left(\lambda^{-\frac{2}{3}} t, \lambda^{-\frac{1}{3}} x\right), \\
Q_{\mu}(x, D) & =P_{\lambda}\left(\lambda^{-\frac{1}{3}} x, D\right) .
\end{aligned}
$$

The function $u_{\mu}(t, \cdot)$ is localized to frequencies of size $\mu$, and the coefficients of $Q_{\mu}$ are localized to frequencies of size less than $\mu^{\frac{1}{2}}$. This implies the following estimates on the coefficients of $Q_{\mu}$ :

$$
\left|\partial_{x}^{\alpha} \mathrm{g}_{\lambda}^{i j}\left(\lambda^{-\frac{1}{3}} x\right)\right|+\left|\partial_{x}^{\alpha} \rho_{\lambda}\left(\lambda^{-\frac{1}{3}} x\right)\right| \leq C_{\alpha} \mu^{\frac{1}{2} \max (0,|\alpha|-2)} .
$$

The interval $I_{\lambda}$ scales to an interval of length $\mu^{-1}$. We have thus reduced the proof of Theorem 1.1 to the following.

Theorem 2.1. Suppose that $u_{\mu}(t, x)$ is localized to frequencies $|\xi| \in\left[\frac{1}{4} \mu, 4 \mu\right]$ and solves

$$
\left(D_{t}+\sum_{1 \leq i, j \leq n} a_{\mu}^{i j}(x) \partial_{x_{i}} \partial_{x_{j}}+\sum_{1 \leq i \leq n} b_{\mu}^{i}(x) \partial_{x_{i}}\right) u_{\mu}=F_{\mu} .
$$

Assume also that the metric satisfies

$$
\begin{gathered}
\left\|a_{\mu}^{i j}-\delta_{i j}\right\|_{C^{2}} \ll 1, \quad\left\|b_{\mu}^{i}\right\|_{C^{1}} \lesssim 1, \\
\operatorname{supp}\left(\widehat{a_{\mu}^{i j}}\right), \operatorname{supp}\left(\widehat{b_{\mu}^{i}}\right) \subset B_{\mu^{1 / 2}}(0) .
\end{gathered}
$$

Then the following estimate holds:

$$
\left.\left\|u_{\mu}\right\|_{L^{p}\left(\left[0, \mu^{-1}\right] ; L^{q}\left(\mathbb{R}^{n}\right)\right.}\right) \lesssim\left\|u_{\mu}\right\|_{L^{\infty}\left(\left[0, \mu^{-1}\right] ; L^{2}\left(\mathbb{R}^{n}\right)\right)}+\left\|F_{\mu}\right\|_{L^{1}\left(\left[0, \mu^{-1}\right] ; L^{2}\left(\mathbb{R}^{n}\right)\right)} .
$$

\section{The PARAmetrix}

We will establish Theorem 2.1 using a short-time wave packet parametrix for the equation (2.5). Wave packet parametrices have been used to establish Strichartz estimates for Schrödinger equations in the work of Staffilani-Tataru [10] and KochTataru [7]; see Tataru [13] for an overview of the methods. The result we need, in fact, is included as a special case in Theorem 2.5 of [7]. The proof of the short time estimate Theorem 2.1 is comparatively simple, though, and therefore we include a self-contained proof here for the reader's benefit.

In this section, then, we use a wave packet transform to construct a parametrix for the operator

$$
D_{t}+A(x, D)+B(x, D)=D_{t}+\sum_{1 \leq i, j \leq n} a_{\mu}^{i j}(x) \partial_{x_{i}} \partial_{x_{j}}+\sum_{1 \leq i \leq n} b_{\mu}^{i}(x) \partial_{x_{i}}
$$

in (2.5) that will yield the Strichartz estimates. For convenience, we suppress the $\mu$ from both the operator and the solution. Let $g$ be a radial Schwartz function 
over $\mathbb{R}^{n}$ such that $\operatorname{supp}(\widehat{g}) \subset B_{1}(0)$ and $\|g\|_{L^{2}}=(2 \pi)^{-\frac{n}{2}}$. For $\mu \geq 1$, we define the operator $T_{\mu}: \mathcal{S}^{\prime}\left(\mathbb{R}^{n}\right) \rightarrow C^{\infty}\left(\mathbb{R}^{2 n}\right)$ by

$$
T_{\mu} f(x, \xi)=\mu^{\frac{n}{4}} \int e^{-i\langle\xi, z-x\rangle} g\left(\mu^{\frac{1}{2}}(z-x)\right) f(z) d z .
$$

$T_{\mu}$ enjoys the property that its adjoint as a map from $L_{x, \xi}^{2}\left(\mathbb{R}^{2 n}\right) \rightarrow L_{z}^{2}\left(\mathbb{R}^{n}\right)$ also serves as a left inverse for $T_{\mu}$, that is, $T_{\mu}^{*} T_{\mu}=I$. This implies that $T_{\mu}$ is an isometry

$$
\left\|T_{\mu} f\right\|_{L_{x, \xi}^{2}\left(\mathbb{R}^{2 n}\right)}=\|f\|_{L_{z}^{2}\left(\mathbb{R}^{n}\right)} .
$$

We conjugate $A(x, D)$ by $T_{\mu}$ and take a suitable approximation to the resulting operator. Specifically, we define the following differential operator over $(x, \xi)$ :

$$
\widetilde{A}=-i d_{\xi} a(x, \xi) \cdot d_{x}+i d_{x} a(x, \xi) \cdot d_{\xi}+a(x, \xi)-\xi \cdot d_{\xi} a(x, \xi) .
$$

By a standard argument from wave packet methods (see for example [12] or [13] where $g$ is Gaussian, or Lemmas 3.1-3.3 in [8] for $g$ as above) we have that if $\widetilde{\beta}_{\mu}$ is a Littlewood-Paley cutoff truncating to frequencies $|\xi| \approx \mu$, then

$$
\left\|T_{\mu} A(\cdot, D) \widetilde{\beta}_{\mu}(D)-\widetilde{A} T_{\mu} \widetilde{\beta}_{\mu}(D)\right\|_{L_{z}^{2} \rightarrow L_{x, \xi}^{2}} \lesssim \mu .
$$

This yields that, if $\tilde{u}(t, x, \xi)=\left(T_{\mu} u_{\mu}(t, \cdot)\right)(x, \xi)$, then $\tilde{u}$ solves the equation

$$
\left(\partial_{t}+d_{\xi} a(x, \xi) \cdot d_{x}-d_{x} a(x, \xi) \cdot d_{\xi}+i a(x, \xi)-i \xi \cdot d_{\xi} a(x, \xi)\right) \tilde{u}(t, x, \xi)=\tilde{G}(t, x, \xi),
$$

where $\tilde{G}$ satisfies

$$
\int_{0}^{\mu^{-1}}\|\tilde{G}(t, x, \xi)\|_{L_{x, \xi}^{2}} d t \lesssim\left\|u_{\mu}\right\|_{L^{\infty}\left(\left[0, \mu^{-1}\right] ; L^{2}\right)}+\left\|F_{\mu}\right\|_{L^{1}\left(\left[0, \mu^{-1}\right] ; L^{2}\right)} .
$$

Given an integral curve $\gamma(r) \in \mathbb{R}_{x, \xi}^{2 n}$ of the vector field

$$
\partial_{t}+d_{\xi} a(x, \xi) \cdot d_{x}-d_{x} a(x, \xi) \cdot d_{\xi}
$$

with $\gamma(t)=(x, \xi)$, we denote $\chi_{s, t}(x, \xi)=\left(x_{s, t}, \xi_{s, t}\right)=\gamma(s)$. Now define

$$
\sigma(x, \xi)=a(x, \xi)-\xi \cdot d_{\xi} a(x, \xi), \quad \psi(t, x, \xi)=\int_{0}^{t} \sigma\left(\chi_{r, t}(x, \xi)\right) d r .
$$

This allows us to write

$$
\tilde{u}(t, x, \xi)=e^{-i \psi(t, x, \xi)} u_{0}\left(\chi_{0, t}(x, \xi)\right)+\int_{0}^{t} e^{-i \psi(t-r, x, \xi)} \tilde{G}\left(r, \chi_{r, t}(x, \xi)\right) d r,
$$

which expresses $\tilde{u}$ as an integrable superposition over $r$ of functions invariant under the flow of $\tilde{A}$, truncated to $t>r$.

Since $u(t, x)=T_{\mu}^{*} \tilde{u}(t, x, \xi)$ it thus suffices to obtain estimates

$$
\left\|\widetilde{\beta}_{\mu}(D) W_{t} f\right\|_{L_{t}^{p} L_{x}^{q}} \lesssim\|f\|_{L_{x, \xi}^{2}}
$$

where $W_{t}$ acts on functions $f(x, \xi)$ by the formula

$$
\left(W_{t} f\right)(y)=T_{\mu}^{*}\left(e^{-i \psi(t, x, \xi)} f\left(\chi_{0, t}(x, \xi)\right)\right)(y) .
$$

By a standard duality argument and an application of the endpoint estimates of Keel and Tao [6] this results from establishing

$$
\begin{gathered}
\left\|\widetilde{\beta}_{\mu} W_{t} W_{s}^{*} \widetilde{\beta}_{\mu}\right\|_{L^{1} \rightarrow L^{\infty}} \lesssim|t-s|^{-\frac{n}{2}}, \\
\left\|\widetilde{\beta}_{\mu} W_{t} W_{s}^{*} \widetilde{\beta}_{\mu}\right\|_{L^{2} \rightarrow L^{2}} \lesssim 1 .
\end{gathered}
$$


The inequality (3.3) follows from the fact that $T_{\mu}$ is an isometry and $\chi_{0, t}(x, \xi)$ is a symplectomorphism, hence preserves the measure $d x d \xi$. The inequality (3.2) is the focus of the next section.

\section{The Dispersive estimate}

In this section, we establish the inequality (3.2). We write the kernel $K(t, y, s, x)$ of $W_{t} W_{s}^{*}$ as

$$
\mu^{\frac{n}{2}} \int e^{-i\langle\zeta, x-z\rangle-i \int_{s}^{t} \sigma\left(\chi_{r, t}(z, \zeta)\right) d r+i\left\langle\zeta_{t, s}, y-z_{t, s}\right\rangle} g\left(\mu^{\frac{1}{2}}\left(y-z_{t, s}\right)\right) g\left(\mu^{\frac{1}{2}}(x-z)\right) d z d \zeta .
$$

Recall that $\operatorname{supp}(\hat{g}) \subset B_{1}(0)$. Since we are concerned with $W_{t} W_{s}^{*} \widetilde{\beta}_{\mu}$, we can insert a cutoff $S_{\mu}(\zeta)$ into the integrand which is supported in a set $|\zeta| \approx \mu$. Note that the Hamiltonian vector field is independent of time, and hence $\chi_{t, s}=\chi_{t-s, 0}$. We drop the zero and abbreviate the latter transformation as $\chi_{t-s}(z, \zeta)=\left(z_{t-s}, \zeta_{t-s}\right)$. It then suffices to consider $s=0$, and we write the kernel $K(t, x, 0, y)$ as

$$
\mu^{\frac{n}{2}} \int e^{-i\langle\zeta, x-z\rangle-i \psi(t, z, \zeta)+i\left\langle\zeta_{t}, y-z_{t}\right\rangle} g\left(\mu^{\frac{1}{2}}\left(y-z_{t}\right)\right) g\left(\mu^{\frac{1}{2}}(x-z)\right) S_{\mu}(\xi) d z d \zeta .
$$

We need to establish uniform bounds over $x$ and $y,|K(t, x, 0, y)| \lesssim t^{-\frac{n}{2}}$. A straightforward estimate shows that

$$
|K(t, x, 0, y)| \lesssim \mu^{n}
$$

meaning that the dispersive estimate holds for $t \leq \mu^{-2}$. We thus assume $t \geq \mu^{-2}$ for the remainder of the section. Lastly we suppose that, in addition, $t \leq \varepsilon \mu^{-1}$ with $\varepsilon$ chosen sufficiently small and independent of $\mu$.

We first need derivative estimates on the transformation $\chi_{t}(z, \zeta)$.

Lemma 4.1. Consider the solutions $\left(z_{t}(z, \zeta), \zeta_{t}(z, \zeta)\right)$ to Hamilton's equations

$$
\partial_{t} z_{t}=d_{\xi} a_{\mu}(z, \zeta), \quad \partial_{t} \zeta_{t}=-d_{x} a_{\mu}(z, \zeta), \quad\left(z_{0}, \zeta_{0}\right)=(z, \zeta) .
$$

We then have the following estimates on the first partial derivatives of $\left(z_{t}, \zeta_{t}\right)$ when $|\zeta| \in\left[\frac{1}{4} \mu, 4 \mu\right]$ and $|t| \leq \mu^{-1}$ :

$$
\begin{array}{cl}
\left|d_{z} z_{t}-I\right| \lesssim \mu t, & \left|d_{\zeta} z_{t}\right| \lesssim t, \\
\left|d_{z} \zeta_{t}\right| \lesssim \mu^{2} t, & \left|d_{\zeta} \zeta_{t}-I\right| \lesssim \mu t, \\
\left|d_{\zeta} z_{t}-\int_{0}^{t}\left(d_{\zeta}^{2} a_{\mu}\right)\left(\chi_{s}(z, \zeta)\right) d s\right| \lesssim \mu t^{2} .
\end{array}
$$

The higher partial derivatives satisfy, for $j+k \geq 2$,

$$
\mu\left|d_{z}^{j} d_{\zeta}^{k} z_{t}\right|+\left|d_{z}^{j} d_{\zeta}^{k} \zeta_{t}\right| \lesssim \mu^{2-k} t\left\langle\mu^{\frac{3}{2}} t\right\rangle^{j+k-1}
$$

Proof. If $|\zeta| \approx 1$, then we can write the Hamilton equations as:

$$
\left(z_{t}, \zeta_{t}\right)=(z, \zeta)+\int_{0}^{t} v\left(z_{s}, \zeta_{s}\right) d s
$$

where the vector field $v$ satisfies

$$
\left|d_{z, \zeta}^{k} v\right| \lesssim \mu^{\frac{1}{2}(k-1)}, \quad k \geq 1 .
$$

Differentiating the equation and using induction yields the bound

$$
\left|d_{z, \zeta}^{k}\left(z_{t}, \zeta_{t}\right)-d_{z, \zeta}^{k}(z, \zeta)\right| \lesssim t\left\langle\mu^{\frac{1}{2}} t\right\rangle^{k-1}, \quad|t| \leq 1
$$


Estimates (4.2) and (4.4) now follow by the rescaling property

$$
\left(z_{t}(z, \zeta), \zeta_{t}(z, \zeta)\right)=\left(z_{\mu t}\left(z, \mu^{-1} \zeta\right), \mu \zeta_{\mu t}\left(z, \mu^{-1} \zeta\right)\right)
$$

Estimate (4.3) follows by differentiating Hamilton's equations as above and applying the bounds (4.2).

We take a partition of unity $\left\{\phi_{m}\right\}_{m \in \mathbb{Z}^{n}}$ over $\mathbb{R}^{n}$ with $\phi_{m}(\zeta)=\phi\left(t^{\frac{1}{2}}\left(\zeta-t^{-\frac{1}{2}} m\right)\right)$ for some $\phi$ smooth and compactly supported. We then write

$$
K(t, y, 0, x)=\sum_{m \in \mathbb{Z}^{n}} K_{m}(t, y, x)
$$

where $K_{m}(t, y, x)$ is defined by

$$
\mu^{\frac{n}{2}} \int e^{-i\langle\zeta, x-z\rangle-i \psi(t, z, \zeta)+i\left\langle\zeta_{t}, y-z_{t}\right\rangle} g\left(\mu^{\frac{1}{2}}\left(y-z_{t}\right)\right) g\left(\mu^{\frac{1}{2}}(x-z)\right) \phi_{m}(\zeta) S_{\mu}(\zeta) d z d \zeta .
$$

The key estimate is that, for $\xi_{m}=t^{-\frac{1}{2}} m$,

$$
\left|K_{m}(t, x, y)\right| \lesssim t^{-\frac{n}{2}}\left(1+t^{-\frac{1}{2}}\left|y-x_{t}\left(x, \xi_{m}\right)\right|\right)^{-N} .
$$

Estimate (4.3), and the fact that

$$
\left\|d_{\xi}^{2} a(x, \xi)-2 I\right\|=2\left\|a^{i j}-\delta^{i j}\right\| \ll 1
$$

yields for $l, m \in \mathbb{Z}^{n}$ and $t \leq \varepsilon \mu^{-1}$,

$$
\left|x_{t}\left(x, \xi_{m}\right)-x_{t}\left(x, \xi_{l}\right)\right| \approx t\left|\xi_{m}-\xi_{l}\right|=t^{\frac{1}{2}}|m-l| .
$$

This now yields

$$
\sum_{m \in \mathbb{Z}^{n}}\left|K_{m}(t, x, y)\right| \lesssim t^{-\frac{n}{2}} \sum_{m \in \mathbb{Z}^{n}}(1+|m|)^{-N}
$$

Since the sum on the right converges for $N$ large this establishes the dispersive estimate.

To prove (4.5), we start by noting that

$$
\partial_{\zeta_{i}}\left(\int_{0}^{t} a\left(z_{r}, \zeta_{r}\right)-\zeta_{r} \cdot\left(d_{\zeta} a\right)\left(z_{r}, \zeta_{r}\right) d r\right)+\zeta_{t} \cdot \partial_{\zeta_{i}} z_{t}=0
$$

The expression vanishes at $t=0$ since $d_{\zeta} z_{0}=0$, and Hamilton's equations show that the derivative of the expression with respect to $t$ vanishes.

As in Theorem 5.4 of Smith and Sogge [9], we now proceed by defining the differential operator

$$
L=\frac{1+i t^{-1}\left(x-z-d_{\zeta} \zeta_{t} \cdot\left(y-z_{t}\right)\right) \cdot d_{\zeta}}{1+t^{-1}\left|x-z-d_{\zeta} \zeta_{t} \cdot\left(y-z_{t}\right)\right|^{2}} .
$$

By the observation above, $L$ preserves the phase function in the definition of $K_{m}$. The estimates (4.2) and (4.4) show that, if $p$ is any one of the functions $\phi_{m}(\zeta)$, $t^{-\frac{1}{2}} z_{t}, \mu^{\frac{1}{2}} z_{t}, S_{\mu}(\zeta), \mu^{-\frac{1}{2}} t^{-\frac{1}{2}} \zeta_{t}$, then for $\mu^{-2} \leq t \leq \mu^{-1}$,

$$
\left|\left(t^{-\frac{1}{2}} \partial_{\zeta}\right)^{k} p\right| \lesssim 1
$$

Integration by parts now yields the following upper bound on $K_{m}(t, x, y)$ :

$$
\begin{aligned}
\mu^{\frac{n}{2}} \int_{\mathbb{R}^{n} \times \operatorname{supp}\left(\phi_{m}\right)}\left(1+t^{-1} \mid(x-z)\right. & \left.-\left.d_{\zeta} \zeta_{t} \cdot\left(y-z_{t}\right)\right|^{2}\right)^{-N} \\
& \times\left(1+\mu^{\frac{1}{2}}|x-z|\right)^{-N}\left(1+\mu^{\frac{1}{2}}\left|y-z_{t}\right|\right)^{N} d z d \zeta
\end{aligned}
$$


We conclude by showing that

$$
t^{-\frac{1}{2}}\left|(x-z)-d_{\zeta} \zeta_{t} \cdot\left(x_{t}-z_{t}\right)\right| \lesssim 1+\mu|x-z|^{2}
$$

where $x_{t}$ denotes $x_{t}\left(x, \xi_{m}\right)$. This implies that the integrand is dominated by

$$
\left(1+t^{-1}\left|d_{\zeta} \zeta_{t} \cdot\left(y-x_{t}\right)\right|^{2}\right)^{-N}\left(1+\mu^{\frac{1}{2}}|x-z|\right)^{-N} .
$$

Since $\left|d_{\zeta} \zeta_{t}-I\right| \lesssim \varepsilon$, this establishes the estimate (4.5), since the $z$ decay and compact $\zeta$ support imply that the integral is essentially over a region in phase space of volume roughly $t^{-\frac{n}{2}} \mu^{-\frac{n}{2}}$.

To establish (4.6), we employ a Taylor expansion and (4.4) to obtain

$$
\begin{aligned}
t^{-\frac{1}{2}} \mid x_{t} & -z_{t}-\left(d_{z} z_{t}\right)(x-z)-\left(d_{\zeta} z_{t}\right)\left(\xi_{m}-\zeta\right) \mid \\
& \lesssim t^{\frac{1}{2}}\left\langle\mu^{\frac{3}{2}} t\right\rangle\left(\mu|x-z|^{2}+|x-z|\left|\xi_{m}-\zeta\right|+\mu^{-1}\left|\xi_{m}-\zeta\right|^{2}\right) \lesssim 1+\mu|x-z|^{2}
\end{aligned}
$$

where the last inequality uses the fact that $\mu^{-2} \leq t \leq \mu^{-1}$ and $\left|\xi_{m}-\zeta\right| \lesssim t^{-\frac{1}{2}}$. In addition, by (4.2),

$$
t^{-\frac{1}{2}}\left|\left(d_{\zeta} z_{t}\right)\left(\xi_{m}-\zeta\right)\right| \lesssim t\left(t^{-\frac{1}{2}}\right)^{2}=1 .
$$

Since $\chi_{t}(z, \zeta)$ is a symplectomorphism, we have

$$
\partial_{\zeta_{i}} \zeta_{t} \cdot \partial_{z_{j}} z_{t}-\partial_{\zeta_{i}} z_{t} \cdot \partial_{z_{j}} \zeta_{t}=\delta_{i j}
$$

where $\cdot$ pairs the $z_{t}$ and $\zeta_{t}$ indices. Lastly, by (4.2),

$$
t^{-\frac{1}{2}}\left|d_{\zeta} z_{t}\right|\left|d_{z} \zeta_{t}\right||x-z| \lesssim \mu^{2} t^{\frac{3}{2}}|x-z| \leq \mu^{\frac{1}{2}}|x-z| .
$$

These facts now combine to yield the estimate (4.6).

\section{REFERENCES}

[1] Anton, R. Strichartz inequalities for Lipschitz metrics on manifolds and the nonlinear Schrödinger equation on domains. Preprint.

[2] Bourgain, J. Fourier transform restriction phenomena for certain lattice subsets and applications to nonlinear evolution equations. I. Schrödinger equations. Geom. Funct. Anal. 3 (1993), no. 2, 107-156. MR1209299 (95d:35160a)

[3] Burq, N., Gérard, P., and Tzvetkov, N. Strichartz inequalities and the nonlinear Schrödinger equation on compact manifolds. Amer. J. Math. 126 (2004), 569-605. MR2058384 (2005h:58036)

[4] Gilbarg D.; Trudinger, N. Elliptic Partial Differential Equations of Second Order. 2nd edition. Springer, New York, 1983. MR737190 (86c:35035)

[5] Ginibre, J.; Velo, G. On the global Cauchy problem for some nonlinear Schrödinger equations. Ann. Inst. H. Poincaré Anal. Non Linéaire 1 (1984), no. 4, 309-323. MR778977 (87a:35164)

[6] Keel, M.; Tao, T. Endpoint Strichartz Estimates. Amer. J. Math 120 (1998), 955-980. MR1646048 (2000d:35018)

[7] Koch, H.; Tataru, D. Dispersive estimates for principally normal operators. Comm. Pure Appl. Math 58 (2005), 217-284. MR2094851 (2005m:35323)

[8] Smith, H.F. Spectral cluster estimates for $C^{1,1}$ metrics. Amer. J. Math., 128 (2006), 10691103. MR2262171

[9] Smith, H.F.; Sogge, C.D. On the $L^{p}$ norm of spectral clusters for compact manifolds with boundary. Acta Math., 198 (2007), 107-153. MR2316270.

[10] Staffilani, G.; Tataru, D. Strichartz estimates for a Schrödinger operator with nonsmooth coefficients. Comm. Partial Differential Equations 27 (2002), no. 7-8, 1337-1372. MR1924470 (2003f:35248)

[11] Strichartz, R. Restriction of Fourier transform to quadratic surfaces and decay of solutions to the wave equation. Duke Math J. 44 (1977), no. 3, 705-714. MR0512086 (58:23577)

[12] Tataru, D. Strichartz estimates for operators with nonsmooth coefficients and the nonlinear wave equation. Amer. J. Math 122 (2000), no. 2, 349-376. MR1749052 (2001c:35260) 
[13] Tataru, D. Phase space transforms and microlocal analysis. Phase space analysis of partial differential equations. Vol. II, 505-524, Pubbl. Cent. Ric. Mat. Ennio Giorgi, Scuola Norm. Sup., Pisa, 2004. MR2208883 (2007e:35309)

[14] M. Taylor, Pseudodifferential Operators and Nonlinear PDE. Progress in Mathematics, vol. 100, Birkhäuser, Boston, 1991. MR1121019 (92j:35193)

Department of Mathematics, Johns Hopkins University, Baltimore, Maryland 21218 E-mail address: mblair@math.jhu.edu

Department of Mathematics, University of Washington, Seattle, Washington 98195 E-mail address: hart@math.washington.edu

Department of Mathematics, Johns Hopkins University, Baltimore, Maryland 21218 E-mail address: sogge@jhu.edu 